\title{
Easy and robust electrotransfection protocol for efficient ectopic gene expression and genome editing in human B-cells
}

Reynand Jay Canoy ${ }^{1,2}$, Franck André ${ }^{1}$, Anna Shmakova ${ }^{1,3}$, Joëlle Wiels ${ }^{1}$, Marc Lipinski ${ }^{1}$, Yegor Vassetzky ${ }^{1,4^{*}}$, Diego Germini ${ }^{1^{*}}$

${ }^{1}$ UMR 9018, CNRS, Univ. Paris-Sud, Université Paris Saclay, Institut Gustave Roussy, 94805

Villejuif, France

${ }^{2}$ Institute of Human Genetics, National Institutes of Health, University of the Philippines Manila, 1000 Manila, Philippines

${ }^{3}$ Laboratory of molecular endocrinology, Institute of Experimental Cardiology, Federal State

Budgetary Organization National Cardiology Research Center Ministry of Health of the Russian Federation, 121552 Moscow, Russia

${ }^{4}$ Koltzov Institute of Developmental Biology, RAS, 117334 Moscow, Russia

*Corresponding authors

Diego Germini germinidiego@gmail.com

Yegor Vassetzky yegor.vassetzky@cnrs.fr 


\section{ABSTRACT}

B-cell lines and primary PBMCs are notoriously hard to transfect, thus making genome editing, ectopic gene expression or gene silencing experiments particularly tedious. Here we propose a novel efficient and reproducible protocol for electrotransfection of lymphoblastoid, B-cell lymphoma, leukaemia cell lines and B-cells from PBMCs. The proposed protocol requires neither costly equipment nor expensive reagents; it can be used with small or large plasmids. Transfection and viability rates of about $79 \%$ and $58 \%$, respectively, have been routinely achieved by optimizing the salt concentration in the electrotransfection medium and the amount of plasmid used. A validation of the protocol was obtained via the generation of a TP53 $^{-/-}$RPMI8866 lymphoblastoid cell line which should prove useful in future hematological and blood cancer studies.

B-cell lines and primary peripheral blood mononuclear cells (PBMCs) are notoriously hard to transfect, thus making genome editing and even simple ectopic gene expression or gene silencing experiments particularly tedious. Cell lines derived from lymphocytes, lymphoma and leukaemia cells belong to the so called hard-to-transfect cells $(1,2)$. Both viral and nonviral (chemical and physical) transfection methods have been tested previously on lymphoid cell lines (3). Retroviral transfection methods allow for relatively high transfection efficiencies, however they carry biosafety risks and international guidelines recommend that they be performed in a biosafety level 2 or 3 laboratory (4). Another concern is that the integration of the genome carried by the virus can produce insertional mutagenesis (5). Vectors based on non-integrating viruses (such as adenovirus or herpesvirus) have shown transfection rates comparable to non-viral methods but they can produce inflammatory-like responses (6).

Electrotransfection is a technique whereby cells are exposed to an electric field which changes the permeability of their membrane allowing the entry of any molecule e.g. drugs, chemicals or nucleic acids (7). This technique was successfully used with different difficult-to-transfect cells (8). Many electrotransfection protocols were described for EBV-transformed lymphoblastoid cell lines (LCLs) and EBV-negative leukaemia and lymphoma cell lines. Most successful protocols involve the use of the Nucleofector II $^{\circledR}$ device (Lonza) which does not allow users to control the parameters of electrotransfection, and result in transfection efficiencies highly variable (10\% to $50 \%$ ) between studies (9-12). Moreover, these rates are 
attained only using small plasmids, the transfection efficiency being much lower when using larger plasmids such as those carrying CRISPR/Cas9. We here report an improved electrotransfection protocol which ensures consistent transfection efficiency with high cell viability using a large variety of lymphoblastoid and malignant B cell-lines and PBMCs.

We used the Cliniporator ${ }^{\mathrm{TM}}$ (Igea) with 1-mm electroporation cuvettes (Cell Projects) and the pCMV-GFP plasmid (3.5 Kbp, PF463, Plasmid Factory) to optimize the electrotransfection parameters for the RPMI8866 LCL. Cells were grown in RPMI 1640 medium supplemented with 10\% FBS, $2 \mathrm{mM}$ L-glutamine, 2\% Glucose, 1mM Sodium pyruvate and 1\% penicillin/streptomycin antibiotics (complete medium, Gibco®, Thermo Fisher Scientific) at $37^{\circ} \mathrm{C}$ in a humidified $5 \% \mathrm{CO}_{2}$ atmosphere. Forty-eight hours before electrotransfection, cells were transferred to the fresh medium to induce them to proliferate and make them more susceptible to transfection (13). On the day of electrotransfection, $5 \times 10^{5}$ cells were resuspended in a total of $100 \mu \mathrm{L}$ electrotransfection mixture composed of the Minimum Essential Medium Eagle with Spinner Modification (S-MEM, Sigma Aldrich), water and plasmid (1 $1 \mu \mathrm{g} / \mu \mathrm{l}$, dissolved in water). The cells were then transferred into $1 \mathrm{~mm}$ cuvettes (Cell Projects) and electrotransfected at $1300 \mathrm{~V} / \mathrm{cm}$, with eight $100 \mu \mathrm{s}$ pulses at $1 \mathrm{~Hz}$. Electrotransfection efficiency and viability rates were evaluated 48 hours after electrotransfection. The viability rate of the electrotransfected cells was calculated with respect to that of non-electrotransfected cells set as $100 \%$ using forward vs. side scatter gating on a BD Accuri ${ }^{\mathrm{TM}}$ C6 Flow Cytometer (BD Biosciences) as shown in the representative FACS plots (Figure 1a). Transfection rates were calculated as percentages of GFP-expressing cells vs. total live cells.

We first tested electrotransfection efficiency and cell viability using the above parameters in

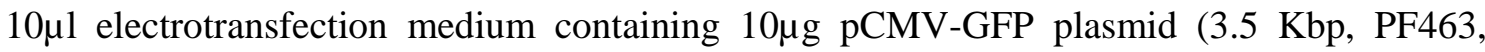
Plasmid Factory) with an addition of $90 \mu \mathrm{l}$ of S-MEM. The electrotransfection efficiency and viability rates (Figure 1bc) did not prove significantly better than other protocols used in previous studies. To improve the transfection rate, the salt concentration of the electrotransfection medium was decreased as reported earlier (14). To do so, the $10 \mu \mathrm{l}$ containing the $10 \mu \mathrm{g}$ of pCMV-GFP plasmid were diluted into 10,40 or $70 \mu \mathrm{L}$ water with 80 , 50, $20 \mu \mathrm{L}$ S-MEM added, respectively, to complete the $100 \mu \mathrm{l}$ volume required for electrotransfection. This resulted in a significantly increased electrotransfection efficiency (up to $73.5 \pm 2.7 \%$ ) but with a gradual decrease in cell viability. Nevertheless, with 50\% S-MEM, 
the transfection rate was as high as $55.3 \pm 2.3 \%$ with a very reasonable cell viability (68 $\pm 3 \%)$ (Figure 1bc).

We next experimented with increased amounts of pCMV-GFP plasmid. Using 50 $\mu$ g rather than $10 \mu \mathrm{g}$ in 50\% water and 50\% S-MEM further improved the electrotransfection efficiency $(79.2 \pm 4.6$ vs. $55.3 \pm 2.3 \%)$ with an only slight decrease in viability $(58.3 \pm 4.8 \%$ vs. $68 \pm 3 \%)$ (Figure 1bc). Altogether, this represents a significant improvement when compared to previously reported protocols.

The described protocol was also suitable for stable expression of the transgene (Supplementary Figure 2). We electrotransfected RPMI-8866 cells with the pCMV-GFP plasmid as described and sorted GFP+ cells after 48 hours. We then sorted cells every seven days during four weeks to obtain a cell population containing 93\% GFP-positive cells.

Applying the protocol thus established to other B-cell lines, namely the Priess and Remb1 LCLs, the BL2 Burkitt lymphoma line, and the RS4;11 acute lymphoblastic leukemia cell line, yielded similar results (transfection efficiency of 41 to 73\%; viability rates of 20 to 55\%) (Figure 1d). We thus suggest that the electrotransfection parameters here defined can be applied successfully to many if not all B-cell lines.

The same electrotransfection protocol was also applied to transfect the pCMV-GFP plasmid into PBMCs purified by Pancoll (PAN biotech) density gradient centrifugation from whole blood samples from healthy donors of different sex and age purchased from the 'Etablissment Français du Sang', Hôpital Saint-Louis, Paris, France in accordance to the French legislation. As the obtained viability and transfection rate were low, we modified the protocol for transfection of PBMCs as follows: we used $400 \mu \mathrm{L}$ of total volume of electrotransfection mixture composed of 50\% SHM medium (19), instead of the S-MEM, in 4 mm cuvettes (Cell Projects); we also increased the electric field to $1500 \mathrm{~V} / \mathrm{cm}$ at 24 pulses. Twenty-four hours after transfection, a $1.5 \pm 0.5 \%$ transfection rate with $30.4 \pm 1.9 \%$ of viability was obtained (Figure 1e). Among the transfected and living cells, $83.7 \pm 3.0 \%$ were CD3+ $\mathrm{T}$ cells and 7.1 $\pm 2.0 \%$, CD19+ B-cells (Supplementary Figure 1).

Most methods currently used for genome editing require the use of large plasmids; unfortunately increasing plasmid sizes negatively affect both transfection efficiency and cell 
viability $(15,16)$. We next tested whether the protocol established for the pCMV-GFP plasmid would also function with a $13.5 \mathrm{Kbp}$ Cas9-GFP plasmid (Addgene \#57818). Electrotransfection of $10 \mu \mathrm{g}$ of the Cas9-GFP plasmid resulted in a good cell viability (64.3 $\pm 4.3 \%)$, but a much lower electrotransfection rate $(1.1 \pm 0.1 \%)$. We then diluted the SMEM medium twofold and used the same electrotransfection parameters adding either $10 \mu \mathrm{g}$ of plasmid and $40 \mu \mathrm{l}$ of water or $50 \mu \mathrm{g}$ of plasmid. While the viability remained virtually unchanged (66.5 $\pm 2.5 \%$ and $61.7 \pm 1.2 \%$, respectively), the transfection rate reached $4.6 \pm 0.8 \%$ when using $50 \mu \mathrm{g}$ of plasmid (Figure 1f). To summarize, cells should be placed in a fresh medium 48 hours before electrotransfection (1300 V/cm, 8 pulses of $100 \mu$ s each, $1 \mathrm{~Hz}$ frequency) performed in 50\% S-MEM and 50\% plasmid diluted in water. This protocol can be adapted to any device that can deliver similar pulses (e.g. the ELECTROcell B10 HVLV (Betatech), the BTX Gemini X2 (BTX Harvard Apparatus) or others) and can be scaled to transfect up to $8 \times 10^{6}$ cells using $50 \mu$ g of plasmid.

We next used the electrotransfection protocol thus optimized to generate TP53 ${ }^{-/}$RPMI 8866 cells. Two gRNAs targeting sequences $7.5 \mathrm{kbp}$ apart in exons 2 and 11 of the TP53 gene were designed (Figure 2a and Supplementary Table 1). They were cloned into the phU6 plasmid (Addgene \#53188) and tested using the previously reported ENIT method (17). RPMI 8866 cells $\left(8 \times 10^{6}\right)$ were then electrotransfected with $30 \mu \mathrm{g}$ Cas9-GFP and $20 \mu \mathrm{g}$ of phU6 plasmids encoding the two gRNAs (10 $\mu$ g/each) in $400 \mu \mathrm{L}$ electrotransfection mixture in 4-mm electroporation cuvettes (Cell Projects). After 48 hours, individual GPF-positive cells were sorted at one cell per well into five 96-well plates, and left to grow in complete medium at $37^{\circ} \mathrm{C}$ in $5 \% \mathrm{CO}_{2}$ atmosphere. Sixty individual clones collected four weeks after transfection were transferred to 24-well plates for clonal expansion. After one further week in culture, the clones were screened using PCR primers covering exons 2 and 11 of the TP53 gene (Supplementary Table 1) and located $8 \mathrm{kbp}$ apart on genomic DNA (Figure 2a). Upon successful deletion of the region, the primer binding sites would be now located $\sim 400$ bp apart. The KO1 and KO2 clones had PCR amplicons of 350 bp and 400 bp (Figure 2b), indicating the successful deletion of a part of p53 gene located between exons 2 and 11.

The two clones expressed neither TP53 mRNA transcripts as tested by qRT-PCR (data not shown) nor the p53 protein as tested by Western Blotting (Figure 2c). That the TP53 gene had been successfully knocked out was confirmed when the expression of the P21 gene, a downstream target of p53 (18), was found significantly reduced as compared to the control 
(Figure 2d). Using the above described method, we have thus been able to successfully and stably knock-out or delete the TP53 gene in a hard-to-transfect B-cell line without using any viral vectors. We believe that the present electrotransfection method will prove instrumental in future cancer research using lymphoma and leukemia B-cell lines and primary blood cells.

\section{Acknowledgements}

This research was supported by grants from the GEFLUC, LNCC, the Presidium of RAS and the State program of fundamental scientific research of IDB RAS. We gratefully acknowledge the technical support of the imaging and cytometry platform of the Gustave Roussy Institute.

\section{Declarations}

The authors declare no conflict of interest. 


\section{References}

1. Zhao N, Qi J, Zeng Z, Parekh P, Chang CC, Tung CH, et al. Transfecting the hard-totransfect lymphoma/leukemia cells using a simple cationic polymer nanocomplex. Journal of Controlled Release. 2012;159(1):104-10.

2. Meacham JM, Durvasula K, Degertekin FL, Fedorov AG. Physical Methods for Intracellular Delivery. Journal of Laboratory Automation. 2014 Feb;19(1):1-18.

3. Kaestner L, Scholz A, Lipp P. Conceptual and technical aspects of transfection and gene delivery. Bioorganic \& Medicinal Chemistry Letters. 2015 Mar;25(6):1171-6.

4. Mosier DE. Introduction for "Safety Considerations for Retroviral Vectors: A Short Review.” Applied Biosafety. 2016;9(2):68-75.

5. Glover DJ, Lipps HJ, Jans DA. Towards safe, non-viral therapeutic gene expression in humans. Nature Reviews Genetics. 2005 Apr 10;6(4):299-310.

6. Kim TK, Eberwine JH. Mammalian cell transfection: The present and the future. Analytical and Bioanalytical Chemistry. 2010;397(8):3173-8.

7. Rols M-P. Electropermeabilization, a physical method for the delivery of therapeutic molecules into cells. Biochimica et Biophysica Acta (BBA) - Biomembranes. 2006 Mar;1758(3):423-8.

8. Jordan ET, Collins M, Terefe J, Ugozzoli L, Rubio T. Optimizing electroporation conditions in primary and other difficult-to-transfect cells. Journal of Biomolecular Techniques. 2008;

9. Chicaybam L, Barcelos C, Peixoto B, Carneiro M, Limia CG, Redondo P, et al. An Efficient Electroporation Protocol for the Genetic Modification of Mammalian Cells. Frontiers in bioengineering and biotechnology. 2016;4:99.

10. Machy P, Lewis F, McMillan L, Jonak ZL. Gene transfer from targeted liposomes to specific lymphoid cells by electroporation. Proceedings of the National Academy of Sciences. 2006;85(21):8027-31.

11. Maurisse R, De Semir D, Emamekhoo H, Bedayat B, Abdolmohammadi A, Parsi H, et al. Comparative transfection of DNA into primary and transformed mammalian cells from different lineages. BMC Biotechnology. 2010;10.

12. Gahn TA, Sugden B. Marked, transient inhibition of expression of the Epstein-Barr virus latent membrane protein gene in Burkitt's lymphoma cell lines by electroporation. J Virol. 1993;67(11):6379-86.

13. Goldstein S, Fordis CM, Howard BH. Enhanced transfection efficiency and improved cell survival after electroporation of G2/M-synchronized cells and treatment with sodium butyrate. Nucleic Acids Research. 1989;17(10):3959-71.

14. Liew A, André FM, Lesueur LL, De Ménorval M-A, O’Brien T, Mir LM. Robust, Efficient, and Practical Electrogene Transfer Method for Human Mesenchymal Stem Cells Using Square Electric Pulses. Human Gene Therapy Methods. 2013 Oct;24(5):289-97.

15. Kreiss P, Cameron B, Rangara R, Mailhe P, Aguerre-Charriol O, Airiau M, et al. Plasmid DNA size does not affect the physicochemical properties of lipoplexes but modulates gene transfer efficiency. Nucleic Acids Research. 1999;27(19):3792-8.

16. Lesueur LL, Mir LM, André FM. Overcoming the Specific Toxicity of Large Plasmids Electrotransfer in Primary Cells In Vitro. Molecular Therapy - Nucleic Acids. 2016;5:e291.

17. Germini D, Saada YB, Tsfasman T, Osina K, Robin CC, Lomov N, et al. A One-Step PCR-Based Assay to Evaluate the Efficiency and Precision of Genomic DNA-Editing Tools. Molecular Therapy - Methods \& Clinical Development. 2017 Jun;5(June):43-50.

18. Georgakilas AG, Martin OA, Bonner WM. p21: A Two-Faced Genome Guardian. Trends in Molecular Medicine. 2017 Apr;23(4):310-9. 
19. Silve A, Leray I, Poignard C, Mir LM. Impact of external medium conductivity on cell membrane electropermeabilization by microsecond and nanosecond electric pulses. Scientific Reports. 2016 Feb 1;6(1):19957. 


\section{Figure legends}

Figure 1. Efficient electrotransfection of human B-cell lines and PBMCs. Effect of different electrotransfection parameters on transfection rate (calculated as GFP expressing cells/living cells, grey) and cell viability (calculated as number of living cells vs. living cells in the untransfected controls, black). Cells were electrotransfected with a pCMV-GFP (a-e) or with a Cas9-GFP plasmids (f) using the Cliniporator ${ }^{\mathrm{TM}}$. Forty eight hours after electrotransfection, the viability and transfection rates were measured using the BD Accuri ${ }^{\mathrm{TM}}$ C6 Flow Cytometer.

(a) Representative cytometry graphs showing the strategy used for gating the viable (upper plots) and transfected (GFP+) (lower plots) LCLs after electrotransfection. Forward scatter area (FSC-A) versus side scatter area (SSC-A) plot was used to gate the cell population and remove the debris. Forward scatter height (FSC-H) vs. area (FSC-A) plot was used to identify the single cell population by choosing a diagonal gating. FITC-channel versus PE-channel plot was used to gate the GFP positive cells where the gating was based on the control cells to ensure $0 \%$ of GFP positive cells in non-electrotransfected control.

(b,c) RPMI8866 cells were electrotransfected at $1300 \mathrm{~V} / \mathrm{cm}, 8$ pulses of $100 \mu$ s length each and $1 \mathrm{~Hz}$ of frequency in the electrotransfection medium composed of the 10-50 $\mu \mathrm{g}$ pCMVGFP plasmid, different amounts of water and S-MEM in a total volume of $100 \mu \mathrm{L}$. Graphs show electrotransfection (b) and viability (c) rates.

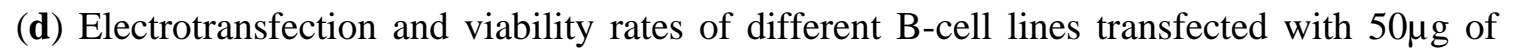
pCMV-GFP plasmid.

(e) Electrotransfection and viability rates of PBMCs transfected with 50 $\mu \mathrm{g}$ of pCMV-GFP plasmid. PBMCs were purified from blood of four different healthy donors by Pancoll (PAN biotech) density gradient centrifugation and electrotransfection and viability rates were tested 24 and 48 hours after electrotransfection.

(f) Effect of plasmid amount and water content on transfection efficiency and viability rates in the RPMI8866 cells transfected with a large Cas9-GFP plasmid.

Every experiment was performed at least in two biological replicates. Data are represented as means \pm SEM. ${ }^{* * *}$ : $\mathrm{P}<0.001$; **: $0.001<\mathrm{P}<0.05 ; *$ : $\mathrm{P}<0.05$, ns: non-significant. Comparisons between more than two averages were performed using the Bonferroni's post-test based on the results generated using the one-way Anova test.

Figure 2. Efficient generation of TP53 $^{-/}$RPMI8866 cells. Electrotransfection was used to 
create RPMI-8866 cells stably knocked-out for the TP53 gene. (a) Gene map showing the designed gRNAs and PCR primers within the TP53 gene. The effective knock-out was confirmed by PCR of genomic DNA (b), western blotting (c) and RT-qPCR for p53 downstream target p21 (d) from one control and two KO lines. Red arrows, gRNAs targeting the exons 2 and 11 of the TP53 gene; blue arrows, the annealing sites of the PCR primers used.

(b) Genomic DNA was amplified using primers annealing next to the exons 2 and 11. M, the molecular weight marker. Successful deletion should generate a PCR amplicon of $\sim 400$ bp in size.

(c) Western blot analysis of the p53 protein. GAPDH was used as a loading control.

(d) Analysis of p21 transcription in two control and two KO RPMI-8866 lines. The transcription of the p21, a direct p53 target, was measured by RT-qPCR. The graph shows fold change vs. the controls after normalization vs. GAPDH.

Every experiment was performed at least in two biological replicates. Data are represented as means \pm SEM. *: $\mathrm{P}<0.05$. Comparisons between more than two averages were performed using the Bonferroni's post-test based on the results generated using the one-way Anova test.

Supplementary Figure 1. Electrotransfection of human PBMCs. A. Representative cytometry graphs showing the gating strategy to determine the viability, transfection rates and percentage of CD3+/CD19+ positive cells after electrotransfection. Forward scatter area (FSC-A) vs. side scatter area (SSC-A) plot was used to gate the cell population and remove the debris. Since PBMCs are not homogenous, we used large gating and eliminated the events with low FSC-A and SSC-A, and those with low FSC-A and high SSC-A to ensure the gating of the majority of viable cells. Forward scatter height (FSC-H) vs. area (FSC-A) plot was used to identify cell populations by choosing a diagonal gating. FITC-channel vs. PE-channel plot was used to gate GFP positive cells, the gating was based on control cells to ensure $0 \%$ of GFP positive cells in the non-electrotransfected control. PE-channel versus APC-channel plot was used to gate CD3+/CD19- T-cells and CD3-/CD19+ B-cells in the total population and in the population of GFP-positive cells after staining with CD19-PE and CD3-APC antibodies. The automatic color compensation on single-stained controls was done by FlowJoTM 10 software (FlowJo LLC, BD, USA). B. PBMCs were electrotransfected at $1500 \mathrm{~V} / \mathrm{cm}, 24$ pulses of $100 \mu \mathrm{s}$ length each and $1 \mathrm{~Hz}$ of frequency in the electrotransfection medium composed of the $50 \mu \mathrm{g}$ pCMV-GFP plasmid, 50\% of water and the SHM medium in a total volume of $400 \mu \mathrm{L}$. T- and B-cell populations were evaluated by CD3-APC and CD19-PE 
staining, respectively, 24 hours after electrotransfection by flow cytometric analysis in the whole population and in the population of transfected (GFP-positive) cells. Experiments were performed in three biological replicates with PBMCs from four different donors. Data are represented as means \pm SEM.

Supplementary Figure 2. Fluorescent photomicrographs of RPMI8866 cells 30 days after electrotransfection with the pCMV-GFP plasmid. GFP+ RPMI8866 were initially sorted 48 hours after electrotransfection, and then were re-sorted every seven days for four weeks. 
Supplementary Table 1. gRNAs and primers sequences.

\begin{tabular}{|c|c|c|}
\hline Function & gRNA/ PCR primers & Sequence (5' to 3') \\
\hline \multirow{2}{*}{$\begin{array}{l}\text { gRNA targeting TP53 } \\
\text { gene exon } 2 \text { and } 11\end{array}$} & p53-ex11 & тСССТСТСССТGTTGGTCGG \\
\hline & p53-ex2 & CTCAGAGGGGGCTCGACGCT \\
\hline \multirow[t]{2}{*}{ PCR primers TP53 DNA } & p53-ex11-F & AAGCGAGACCCAGTCTCAAA \\
\hline & p53-ex2-R & CAGCCATTCTTTTCСТGCTC \\
\hline \multirow{2}{*}{$\begin{array}{ll}\text { PCR primers } & \text { GAPDH } \\
\text { DNA } & \end{array}$} & GAPDH DNA-F & GCTGGCACCACTACTTCAGAG \\
\hline & GAPDH DNA-F & $\begin{array}{l}\text { GCCAACAGCAGATAGCCTAG } \\
\text { G }\end{array}$ \\
\hline \multirow{2}{*}{ PCR primes $p 21$ mRNA } & P21-F & CTGCCGAAGTCAGTTCCTTGT \\
\hline & P21-R & CATGGGTTCTGACGGACATC \\
\hline \multirow{2}{*}{$\begin{array}{l}\text { PCR primes GAPDH } \\
\text { mRNA }\end{array}$} & GAPDH mRNA-F & CTGCACCACCAACTGCTTAG \\
\hline & GAPDH mRNA-F & AGGTCCACCACTGACACGTT \\
\hline
\end{tabular}


A
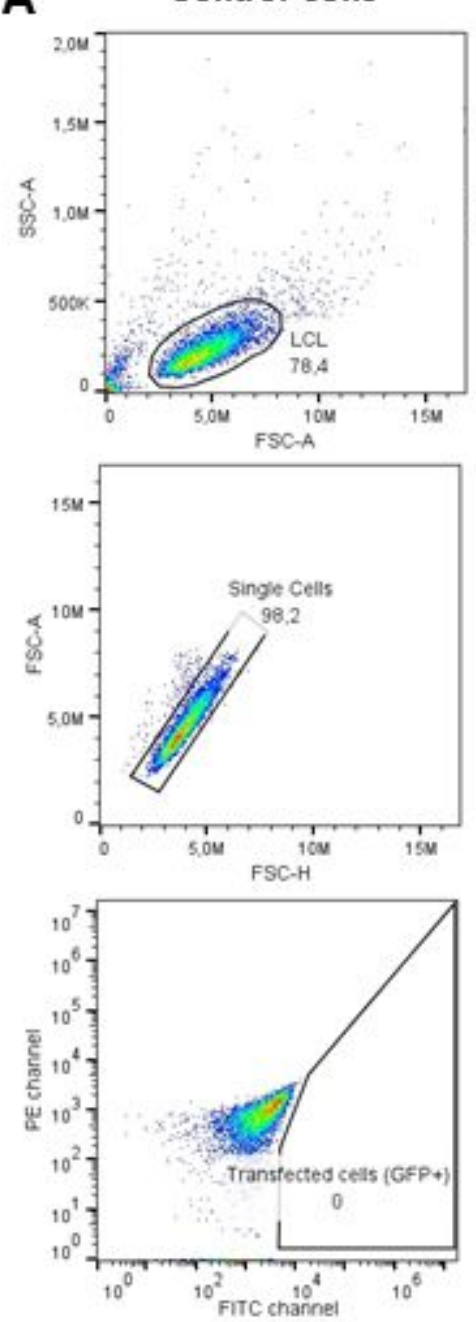

E

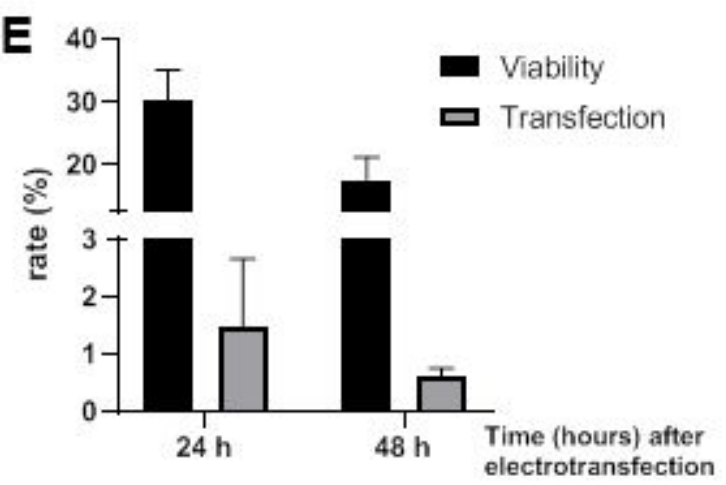

Electrotransfected cells
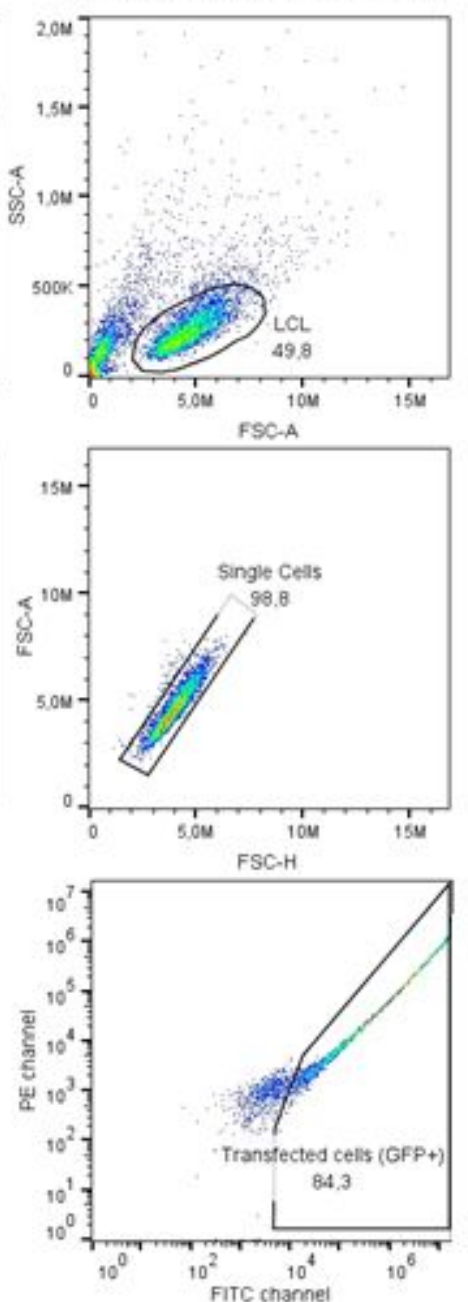

$F$

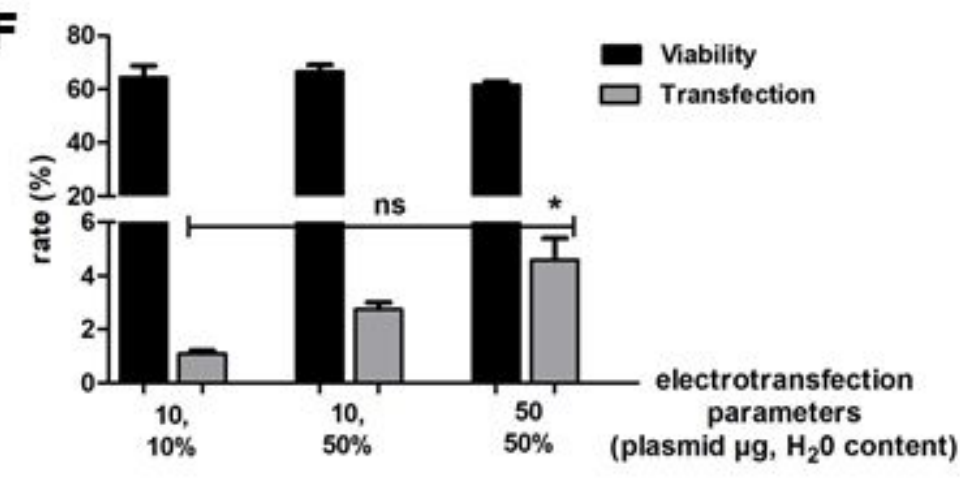

Figure 1 


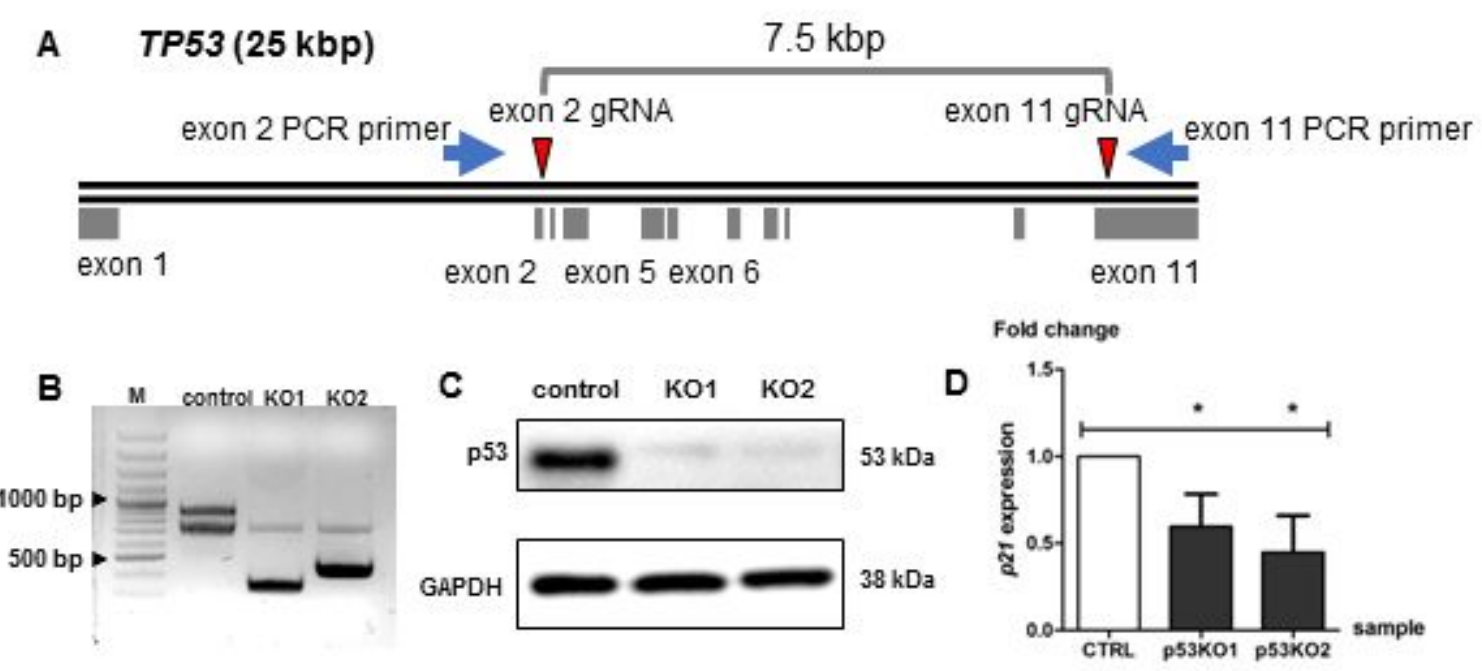

Figure 2 